\title{
Should women at high risk of neural tube defect have an amniocentesis?
}

\author{
K M LAURENCE*, G ELDER $†, K$ T EVANS $\ddagger$, B M HIBBARD \\ M HOOLE $\S$, AND C J ROBERTS\|
}

From the Departments of ${ }^{*}$ Child Health, $\nmid$ Medical Biochemistry, $\ddagger$ Diagnostic Radiology, $\$$ Obstetrics and Gynaecology, and $\|$ Community Medicine, University of Wales College of Medicine, Heath Park, Cardiff CF4 $4 X N$.

SUMMARY As part of an investigation into the practical problems of a maternal serum alphafetoprotein (AFP) neural tube defect (NTD) screening programme carried out in Mid Glamorgan, South Wales, between 1977 and 1979, obstetricians were recommended to refer women with high risk pregnancies directly for counselling, high resolution ultrasonography, and amniocentesis without first carrying out serum screening. Out of 15687 pregnant women onethird attended too late to be screened. A total of 637 was classed as high risk, mostly at greater risk than 1 in 50 because of a previously affected pregnancy or an affected close relative. Compliance with recommended procedure was relatively low as many were screened. There were 10 pregnancies with a recurrence of NTD, of which one was not tested at all, two were not detected (one closed meningocele and one closed iniencephalic), and seven were detected and the pregnancies terminated. All the latter, as well as the iniencephalic, would have been detected from a serum AFP determination and a high resolution ultrasound scan alone. It is concluded that these investigations are sufficient for high risk pregnancies and that amniocentesis is not really cost effective or necessary unless either of these investigations is abnormal. As numbers in this study were small it is suggested that these conclusions should be tested in a larger study.

Prenatal diagnosis of neural tube defects following an ultrasound scan (USS) and amniocentesis has for over a decade been part of antenatal care for women at increased risk for NTD. ${ }^{1}$ Screening for these defects had become a possibility following the publication of the UK Collaborative Study in $1977^{2}$ which suggested that $88 \%$ of open NTD would be detected at 16 to 18 weeks' gestation if 2.5 multiples above the median was used as the cut off point for maternal serum AFP. Since the publication of the report of the working party for screening neural tube defects (the Black Report), ${ }^{3}$ population screening for NTD has been increasingly undertaken in the United Kingdom.

In 1976 the Department of Health and Social Security and the Welsh Office provided funding for an interdisciplinary South Wales Anencephaly and Spina Bifida Study Group to examine the acceptability, effectiveness, and operational issues of maternal serum AFP screening under field conditions. ${ }^{4}$ In

Received for publication 15 July 1985. Accepted for publication 13 August 1985. this investigation, obstetricians were also given the option of referring for prenatal diagnosis women known to be at increased risk for NTD ${ }^{5}$ because of a family history without first having serum AFP screening. This paper describes how this policy was followed in practice and how effective it turned out to be.

\section{Methods}

The investigation was mounted between January 1977 and August 1979 in Mid Glamorgan, a nonteaching Health Authority with a population of 540000 , and at the time of the study with a high prevalence $(4 \cdot 7 / 1000$ births) of NTD. Included in the study were 15687 women with complete information who were booked for confinement in the National Health Service maternity units $(98.6 \%$ of all pregnancies in the area). Clinic midwives working with eight consultants in seven clinics identified the following groups of high risk mothers.

(1) Mothers who had previously had one or more pregnancies with a NTD. 
(2) Fathers who in a previous marriage or liaison had a child with a NTD.

(3) Parents who themselves had spina bifida.

(4) Parents with sibs who had NTD.

(5) Parents whose sib had a pregnancy with a NTD or where their parents, aunts, or uncles had a NTD.

It was recommended that subjects in these groups should be referred for genetic counselling and high resolution USS $^{67}$ to a central assessment clinic organisation set up as part of the screening programme. This was followed in some cases with amniocentesis at 15 weeks or as soon as possible thereafter, without measuring maternal serum AFP. In the majority of cases referred to the central assessment clinic a blood sample for serum AFP estimation was also taken just before amniocentesis, but this was for research purposes and was not considered part of the screening procedure. For the last year of the study women in group 5 were no longer referred directly as the climate of opinion had changed about amniocentesis for this group (table 1). Women with no family history of NTD had a blood sample taken for serum AFP estimation between 16 and 19 weeks. Those first attending after 19 weeks were considered to be too late for screening.

Obstetricians were not obliged to adhere to these recommended procedures and were at liberty to decide on their own policies, provided that these were known to the study group.

\section{Results}

In the Mid Glamorgan population of 15687 pregnant women, there were 73 who had a pregnancy with NTD: 39 with anencephaly (or one of its variants) and 34 with spina bifida, seven of which were closed lesions. This is a prevalence of 4.7 per 1000 pregnancies that had reached 16 weeks' gestation $(2 \cdot 5$ per 1000 for anencephaly and $2 \cdot 2$ per 1000 for spina bifida).

The accuracy with which women at high risk for NTD were identified by the antenatal clinical staff

TABLE 1 Risk for neural tube malformation*.

\begin{tabular}{ll}
\hline Risk situation & Approximate risk \\
\hline Whole population & $1 / 200$ \\
One previous affected child & $1 / 20$ \\
Two previous affected children & $1 / 10$ \\
One parent affected & $1 / 25$ \\
One aunt or uncle affected & $1 / 50$ \\
One cousin or great-aunt or uncle affected & $1 / 100$ \\
Other relatives affected & $1 / 200$
\end{tabular}

*Based on a family study carried out in South Wales between 1956 and $1962 .{ }^{5}$ was tested by a random 1 in 10 check carried out by a Research Midwifery Officer in 1978. In 367 booking interviews the clinic midwife correctly identified 10 women with a previous obstetric or family history of NTD (three first, one second, and six third degree relatives, and three more with distantly related family members). They misclassified one with a third degree relative (a microcephalic) and missed one with a distant relative with NTD.

Altogether 637 women at high risk for NTD were identified among the 15687 pregnant women in Mid Glamorgan, $4 \cdot 1 \%$ of the total. These included 155 at a risk of 1 in 100 or less and $482(3.0 \%)$ at a risk of 1 in 50 or greater. The latter were divided approximately equally between those who had a risk of about 1 in 50 and those who had a risk of 1 in 20 or greater.

A total of 103 high risk women had neither routine nor high risk surveillance, 69 of them because they attended antenatal clinic too late. Of the other 34 , for only eight of these is there a record of a definite refusal. Out of the 103 who were not investigated, 13 women had a 1 in 20 or greater risk; one of them did not book until 32 weeks and gave birth to an infant with open spina bifida who survived but was severely handicapped (table 2).

In only 72 instances was the recommended high risk policy of 'direct to USS and amniocentesis' strictly adhered to, including six women who first attended after the 19th week. Among these, one anencephalic pregnancy which was terminated was identified from both an abnormal USS and an abnormal amniotic fluid AFP level.

A total of 454 high risk women went through the maternal serum AFP screening procedure. Of these, 218 had no further investigations after a normal serum AFP was reported. However, among these was one woman who had a pregnancy with NTD. She had a normal serum AFP level at 18 weeks but then developed polyhydramnios at 32 weeks, had gross fetal abnormality on USS, and delivered an iniencephalic fetus with a closed lesion. One other woman had a grossly raised serum AFP level at 18 weeks which led to the termination of the pregnancy with the delivery of a fetus with a craniorachischisis.

A total of 156 women was referred for genetic counselling, USS, and amniocentesis after serum AFP screening had first been carried out. There were three pregnancies with NTD. In one anencephalic pregnancy the serum AFP level was interpreted as being within normal limits for 16 weeks, but the gestational dating was subsequently shown to have been only 12 weeks. USS, preamniocentesis serum, and the amniotic AFP levels were all grossly abnormal at 16 weeks and the pregnancy was terminated. The second anencepha- 
TABLE 2 High risk for neural tube defect at booking.

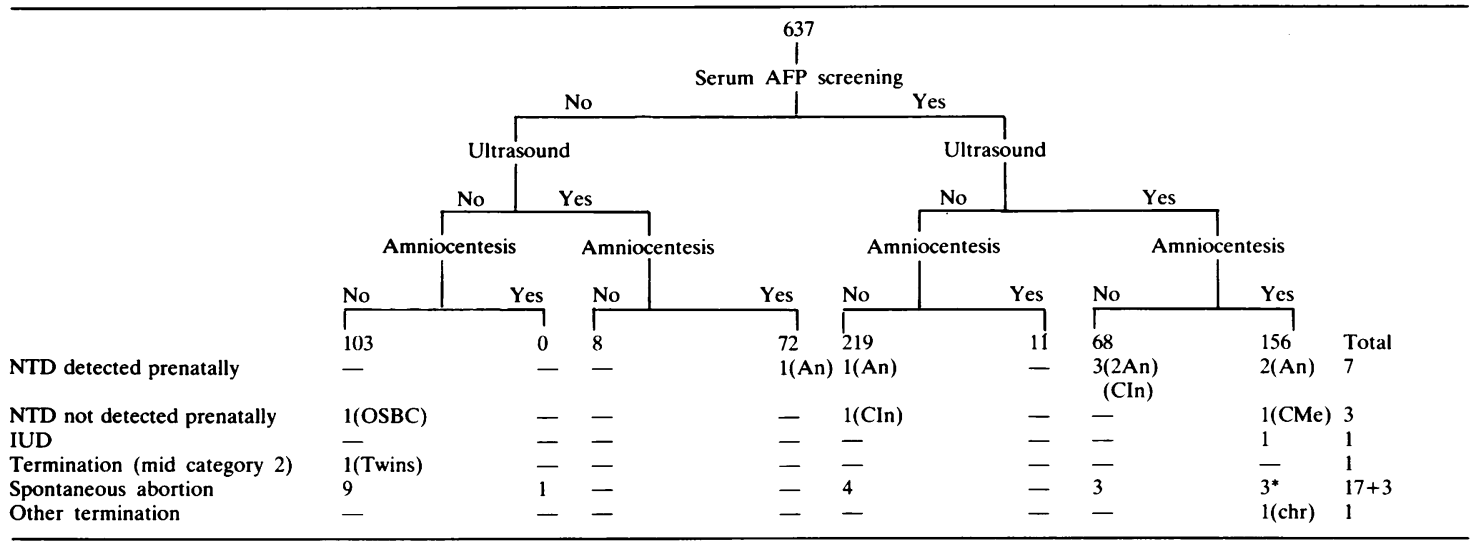

*Within one month of amniocentesis.

$(\mathrm{OSBC})=$ open spina bifida

(An) = ancephaly.

(CIn) =closed iniencephaly

$(\mathrm{CMe})=$ closed meningocele

(chr) = chromosome abnormalities .

lic pregnancy was terminated where a serum AFP taken at 15 weeks was normal, but a USS, the preamniocentesis serum, and the amniotic AFP level two weeks later were all abnormal. The third pregnancy went to term with the birth of an infant with a closed meningocele, who after neonatal surgery survived virtually without handicap. The serum AFP level thought to be abnormal, owing to underestimation of the gestational age, was followed by a normal USS, pre-amniocentesis serum, and amniotic AFP level.

Seventy-six pregnancies, all but eight having had maternal serum AFP screening first, were monitored with USS but not with an amniocentesis. Two anencephalic pregnancies were terminated, both with an abnormal serum AFP level and an abnormal USS. In one other woman, with a normal serum AFP level at 16 weeks and an abnormal USS, the pregnancy was terminated on the findings of the latter alone, with the delivery of an iniencephalic with a closed lesion.

Finally, 11 pregnancies were monitored with an amniocentesis without previous USS by a local obstetrician who had no USS facilities at the beginning of the screening programme.

\section{Discussion}

Ten NTD occurred in women identified as being at increased risk (all at risk of 1 in 20 or greater), suggesting that in the Mid Glamorgan population six out of seven NTD occurred in women with no previous obstetric history of NTD or close relative affected.

In two of the high risk pregnancies with NTD, serum AFP estimation was carried out too soon, at 12 weeks because of mistaken gestational dating in one, and at 15 weeks in the other. The anencephaly would have remained undetected had the high risk option of high resolution USS and amniocentesis not been available. The same would have applied to a pregnancy of a closed iniencephaly where the serum AFP level was normal at 16 weeks. Had maternal serum AFP screening been carried out properly, no open NTD in a high risk woman would have been missed. With high resolution USS as well as maternal serum AFP screening, only the closed meningocele would have remained undetected, and this situation would not have changed with the addition of amniocentesis.

The compliance with the recommended procedure for high risk mothers was low (12\%), making it difficult to evaluate the effectiveness of the option on its own. In practice, obstetricians were often reluctant to challenge high risk pregnancies, many of which in their experience would subsequently turn out to be normal. Furthermore, there was good reason to believe that the sensitivity was high, ${ }^{2}$ so they preferred instead to take a blood sample and wait for the result. In addition, many women had their serum AFP taken as part of the screening programme and were then sent for amniocentesis 
even though the serum AFP level was within normal limits. Further, a proportion had the USS but no amniocentesis, in most instances after a serum AFP. Of the 637 high risk mothers, $540(85 \%)$ booked at antenatal clinics by 19 weeks' gestation and could therefore have been eligible for maternal serum AFP screening. In fact, 454 were so screened. Less than half (304) had a high resolution USS and only just over one-third (239) had an amniocentesis.

The increased financial cost of the high risk option is not great. ${ }^{8}$ However, the $98 \%$ of mothers tested who will not have a detectable NTD will have experienced a good deal of unnecessary anxiety while waiting for the USS and amniocentesis results. ${ }^{9-11}$ In addition, there will be some consequential abortions, as even in the most competent and practised hands amniocentesis carries a risk of about 1 in $200 .^{12}$ The benefit of the high risk procedure is that some women, knowing themselves to be at increased risk for NTD, will feel less anxious about the outcome of the pregnancy, in the knowledge that tests had been performed which would detect very nearly all open NTD, rather than relying on a test which could leave as many as one-fifth of pregnancies with open spina bifida undetected. ${ }^{2}$ As amniocentesis can be carried out as early as the 15th week of gestation, parents are likely to have reassurance about NTD up to four weeks earlier than if they relied on the serum screening test, followed perhaps by a high precision USS and amniocentesis. They will also have the benefit of a consultation with a clinical geneticist which many will not previously have had. ${ }^{13}$ Finally, some other conditions, such as abdominal wall defect and chromosome abnormality, may be detected at the same time by USS or amniocentesis. With the improving technical quality of scanning, other conditions such as closed encephalocele, hydrocephalus, severe bone dysplasia, and other abnormalities may also be identified.

The number of cases in this study was very small. However, it seems that all the cases of NTD, with the exception of the closed meningocele, that occurred in the high risk women would have been detected by a combination of serum AFP screening and high resolution USS, with the latter at least alerting the obstetrician to errors in the estimation of gestational age and to anencephaly and its variants. It appears that offering a service for pregnancies at high risk for NTD consisting of both high resolution USS and amniocentesis may not be really cost effective when compared with the relative success of a properly carried out maternal serum AFP screening programme. We suggest that the full high resolution USS and amniocentesis option is unnecessary, except for those women at particularly high risk (in groups 1,2, and 3) who specifically request amniocentesis, or where parental anxiety is high for other reasons. The normal procedure should therefore be a maternal serum AFP estimation combined with a high resolution USS at 16 to 18 weeks' gestation, as suggested by Harris and Read, ${ }^{14}$ especially as the latter investigation has improved greatly since $1979^{7}$ and is likely to become very much more sophisticated in the future. An amniocentesis for amniotic AFP estimation and identification of isoenzymes of acetylcholinesterase probably should only be carried out if either the serum AFP level is raised or the USS is suspicious or abnormal. However, a larger study to test this approach should be carried out urgently.

We thank the Members and Officials of Mid Glamorgan Health Authority for their support and the many Consultant Obstetricians and other medical, nursing, technical, and clerical staff for their help. Financial support was provided by the Department of Health and Social Security and the Welsh Office.

\section{References}

1 Laurence KM. Genetics and prevention of neural tube defects. In: Emery AEH, Rimoin DL, eds. Principles and practice of medical genetics. Vol 1. Edinburgh: Churchill Livingstone, 1983: 231-45.

2 UK Collaborative Study on Alpha-fetoprotein in Relation to Neural Tube Defects. Maternal serum alpha-fetoprotein measurement in antenatal screening for anencephaly and spina bifida in early pregnancy. Lancet 1977;i:1323-32.

3 The Working Group on Screening for Neural Tube Defects. Report by the working group on screening for neural tube defects. (Black report.) London: DHSS, 1979.

4 Roberts CJ, Hibbard BM, Elder GH, et al. The efficacy of a serum screening service for neural tube defects; the South Wales experience. Lancet 1983;i:1315-8.

5 Carter CO, David PA, Laurence KM. A family study of central nervous system malformations in South Wales. J Med Genet 1968;5:81-106.

6 Roberts CJ, Hibbard BM, Evans DR, et al. Precision in estimating gestational age and its influence on sensitivity of alpha-fetoprotein screening. Br Med J 1979;i:981-3.

7 Roberts CJ, Evans KT, Hibbard BM, Laurence KM, Roberts EE, Robertson IB. Diagnostic effectiveness of ultrasound in detection of neural tube defect: the South Wales experience of 2509 scans (1977-1982) in high risk mothers. Lancet 1983;ii:1068-9.

${ }^{8}$ Hibbard BM, Roberts CJ, Elder GH, Evans KT, Laurence KM. Can we afford screening for neural tube defects? The South Wales experience. Br Med J 1985;290:293-5.

9 Fearn J, Hibbard BM, Laurence KM, Roberts A, Robinson JO. Screening for neural tube defects and maternal anxiety. $\mathrm{Br} J$ Obstet Gynaecol 1982;89:218-21.

10 Robinson JO, Hibbard BM, Laurence KM. Anxiety during a crisis: emotional effects of screening for neural tube defects. $J$ Psychol Res 1984;28:163-9. 
$"$ Laurence KM, Morris J. The effect of the introduction of prenatal diagnosis on the reproductive history of women at increased risk for neural tube defects. Prenatal Diagnosis 1981;1:51-60.

12 Roberts A. Report of the South Wales Anencephaly and Spina Bifida Study Group, 1981 (unpublished).

${ }^{13}$ Carter J, Laurence KM. Genetic counselling during pregnancy for couples at high risk for neural tube defect: is this the optimum time to attend? Biol Soc 1984;1:185-92.
${ }^{14}$ Harris R, Read AP. New uncertainties in prenatal screening for neural tube defect. Br Med J 1981;282:1416-8.

Correspondence and requests for reprints to Professor K M Laurence, Department of Child Health, University of Wales College of Medicine, Heath Park, Cardiff CF4 4XN. 